\section{Correlation of central venous and pulmonary capillary wedge pressures}

To the Editor:

The study by Gallacher et al addresses a most important question concerned with the perioperative management of patients undergoing aortic surgery and indeed is relevant to all patients undrigoing major surgery. Is there any preoperative, non-invasive investigation that will help us determine those patients which might benefit from the use of a pulmonary artery catheter during surgery and in the immediate postoperative period? If we can demonstrate good correlation between the central venous pressure and pulmonary wedge pressure in certain sub-groups of patients, then the need to monitor the filling pressure of both ventricles might be obviated. We could perhaps in some circumstances infer the filling pressure of the left ventricle from the knowledge of the central venous pressure and the complications and cost of pulmonary artery catheterization would be avoided.

Inherent in the discussion of this question is a consistent definition of correlation. Regression analysis is a respected, statistical tool that is in common use in the medical literature and I see no purpose in introducing a "homemade" definition of correlation such as that used in the paper by Gallacher et al. The central venous pressure was considered not to correlate with pulmonary capillary wedge pressure if a difference of five $\mathrm{mmHg}$ was present. It would be churlish to belabour the point but clearly all conclusions based on this fundamental error must be regarded with the greatest suspicion. I would be interested to hear why the authors opted for such an arbitrary definition.

David G. Whalley MB FFARCS FRCP(C)

Department of Anaesthesia

Royal Victoria Hospital and McGill University Montreal, Quebec
REFERENCE

1 Gallacher WN, Goldberg I, Houston P, Druck M, Dunnington $S$. The value of nuclear angiography in the preoperative assessment of patients undergoing elective aortic surgery. Can Anaesth Soc I 1984, 31: 523-7.

REPLY

$I$ would like to respond to Dr. Whalley's criticism by describing the reason why we adopted our definition of correlation between $C V P$ and $P C W P$.

Our study was essentially clinical in nature and the terms we used were clinical for that reason. The aim of the study was to evaluate how preoperative nuclear angiography might modify our current management of patients undergoing aortic surgery. All patients had preoperative nuclear angiography which provided information about ejection fraction $(E F)$ and wall morion $a b$ normalities. In addition, we evaludsed all patients on a clinical basis. If the patients had clinical evidence or history of ischaemic heart disease or heart failure we considered these as indications for a pulmonary artery (PA) line.

We wanted to know if we were missing patients who should have this monitoring. The rype of patient we were looking for would have no clinical history or physical findings which would cause us to monitor PCWP but would have an abnormal nuclear scan. The intraoperative course of each patient was the factor which decided whether in the ultimate analysis the PA line had been necessary or not.

We knew from studies by Nicholas et al.' and Geiman et al. ${ }^{2}$ that in patients undergoing aortic surgery the correlation overall between CVP and PCWP was good. Nicholas et al. showed a correlation coefficient $(r)$ between CVP and PCWP of $0.68(p<0.01)$. The relationship was given by $C V P=2.28+0.5 / P C W P$. Gelman et al. showed that the correlation coefficient $(r)$ between $C V P$ and $P C W P$ values for 13 patients undergoing aortic surgery was $0.69(p<0.05)$. The relationship was given by $C V P=-1.04+0.70$ PCWP.

Relationships between changes in CVP ( $\triangle C V P$ ) and changes in PCWP ( $\triangle P C W P$ ) were somewhat stronger $r=0.73(p<0.01) \Delta C V P=0.37+0.59 \triangle P C W P$ for all patients. Gelman's conclusion was "although CVP accurately predicted direction and magnitude of PAOP in the majority of patients. there may be some of them in whom therapy based on CVP might be detrimental."

Our definition was designed to pick out those individuals referred to by Gelman et al. i.e. those whose management is altered by knowledge of the PCWP. Our definition puts emphasis on the numerical value of the CVP and PCWP. Take for example one of our patients whose $E F$ was 28 per cent. The correlation coefficient for this individual was 0.93. $(p<0.01)$. If one looks at the raw data his CVP was markedly lower than PCWP 